\title{
On the Asymptotic Growth of Entire Monogenic Functions
}

\author{
R. de Almeida and R. S. Kraußhar
}

\begin{abstract}
In this paper we analyze the behavior of growth of entire monogenic functions in higher dimensional Euclidean spaces. Generalizations of growth orders, the maximum term and of the central index to Clifford analysis provide the basic tools for our analysis. We obtain generalizations of some Valiron's inequalities for transcendental entire monogenic functions. Further to this an asymptotic relation between the growth of a monogenic function and their iterated radial derivatives is established.
\end{abstract}

Keywords: Monogenic functions, asymptotic growth, maximum term, central index, Valiron's inequalities, partial differential equations

MSC 2000: Primary 30G35, secondary 30D15

\section{Introduction}

In one variable complex analysis much effort has been done in the study of the asymptotic growth of holomorphic and meromorphic functions during the last century, starting for example with the famous works of A. Wiman [17], G. Valiron [16], R. Nevanlinna [15] their students, and many others. Their asymptotic analysis provided powerful tools to study complex partial differential equations (see, e.g., $[11,10]$ among many others), so that this research domain has emerged to be a field of central and vast interest within complex function theory.

Regina de Almeida: Departamento de Matemática, Universidade de Trás-os-Montes e Alto Douro, P-5000-911 Vila Real, Portugal; Lehrstuhl II für Mathematik, RheinischWestfälische Technische Hochschule Aachen, D-52056 Aachen, Germany;

ralmeida@utad.pt

Partial financial support from the grant ProdepIII (2/5.3/2001) from FSE and from FCT (SFRH/BD/8330/2002) gratefully acknowledged

Rolf Sören Kraußhar: Department of Mathematical Analysis, Ghent University, Building S-22, Galglaan 2, B-9000 Ghent, Belgium; krauss@cage.UGent. be

ISSN 0232-2064 / \$2.50 C Heldermann Verlag Berlin 
Very quickly one observed that a number of the classical results carry over relatively easily to the framework of several complex variables, in particular many central parts of Wiman's and Valiron's theory, as illustrated for instance in the textbook [10].

Another higher dimensional generalization of classical complex analysis is Clifford analysis, which has been recognized to be a valuable counterpart to several complex variables theory for tackling higher dimensional problems during the last decades. Clifford analysis considers Clifford algebra valued functions defined in open subsets of $\mathbb{R}^{n+1}$ that satisfy higher dimensional generalizations of the Cauchy-Riemann system. This approach opened the door to carry over a number of classical and powerful theorems from one variable complex analysis to higher dimensions, such as for instance a Cauchy integral formula, an argument principle and the whole residue and singularity theory. See for instance $[2,5,9,12]$ among many other important contributions that point in this direction.

However, as far as we know, relatively little effort has been done to establish analogies of the above indicated asymptotic results within the context of Clifford analysis so far. In the very recent paper [1] some results concerning the asymptotic growth of a particular subclass of Clifford holomorphic functions that are built as series from a special family of particular polynomials have been established. However, as the main intention in [1] was to describe certain special Cannon sets of that special family of Clifford algebra valued functions, many of the central questions concerning the asymptotic analysis remained untouched.

The aim of this paper is to fill in some of these gaps and to establish some very first rudiments of a generalized Wiman-Valiron theory in the context of Clifford analysis. Generalizations of growth orders, the maximum term and of the central index to Clifford analysis provide the basic tools for our analysis. We obtain generalizations of some Valiron's inequalities for transcendental entire monogenic functions. Further to this an asymptotic relation between the maximum term of a Clifford holomorphic function and that of their iterated radial derivatives will be established.

\section{Preliminaries}

We begin by introducing the basic notions and concepts. For detailed information about Clifford algebras and their function theory we refer, for example, to $[2,5]$ and $[9]$.

2.1. Clifford algebras. By $\left\{e_{1}, e_{2}, \ldots, e_{n}\right\}$ we denote the canonical basis of the Euclidean vector space $\mathbb{R}^{n}$. The attached real Clifford algebra $\mathbf{C l}_{0 n}$ is the free algebra generated by $\mathbb{R}^{n}$ modulo the relation $\mathbf{x}^{2}=-\|\mathbf{x}\|^{2} e_{0}$, where $\mathbf{x} \in \mathbb{R}^{n}$ and 
$e_{0}$ is the neutral element with respect to multiplication of the Clifford algebra $\mathbf{C l}_{0 n}$. In the Clifford algebra $\mathbf{C l}_{0 n}$ the following multiplication rules hold:

$$
e_{i} e_{j}+e_{j} e_{i}=-2 \delta_{i j} e_{0}, \quad i, j=1, \cdots, n,
$$

where $\delta_{i j}$ is the Kronecker symbol. A basis for the Clifford algebra $\mathbf{C l}_{0 n}$ is given by the set $\left\{e_{A}: A \subseteq\{1, \cdots, n\}\right\}$ with $e_{A}=e_{l_{1}} e_{l_{2}} \cdots e_{l_{r}}$, where $1 \leq l_{1}<\ldots$ $<l_{r} \leq n, e_{\emptyset}=e_{0}=1$. Each $a \in \mathbf{C l}_{0 n}$ can be written in the form $a=\sum_{A} a_{A} e_{A}$ with $a_{A} \in \mathbb{R}$. Two examples of real Clifford algebras are the complex number field $\mathbb{C}$ and the Hamiltonian skew field $\mathbb{H}$.

The conjugation anti-automorphism in the Clifford algebra $\mathbf{C l}_{0 n}$ is defined by $\bar{a}=\sum_{A} a_{A} \bar{e}_{A}$, where $\bar{e}_{A}=\bar{e}_{l_{r}} \bar{e}_{l_{r-1}} \cdots \bar{e}_{l_{1}}$ and $\bar{e}_{j}=-e_{j}$ for $j=1, \cdots, n, \bar{e}_{0}=$ $e_{0}=1$. The linear subspace $\operatorname{span}_{\mathbb{R}}\left\{1, e_{1}, \ldots, e_{n}\right\}=\mathbb{R} \oplus \mathbb{R}^{n} \subset \mathbf{C l}_{0 n}$ is the socalled space of paravectors $z=x_{0}+x_{1} e_{1}+x_{2} e_{2}+\cdots+x_{n} e_{n}$ which we simply identify with $\mathbb{R}^{n+1} . x_{0}=: \operatorname{Sc}(z)$ is called the scalar part of the paravector $z$ and $\mathbf{x}:=x_{1} e_{1}+\cdots+x_{n} e_{n}=: \operatorname{Vec}(z)$ its vector part.

A scalar product between two Clifford numbers $a, b \in \mathbf{C l}_{0 n}$ is defined by $\langle a, b\rangle:=\operatorname{Sc}(a \bar{b})$ and the Clifford norm of an arbitrary $a=\sum_{A} a_{A} e_{A}$ is $\|a\|=$ $\left(\sum_{A}\left|a_{A}\right|^{2}\right)^{\frac{1}{2}}$. Any paravector $z \in \mathbb{R}^{n+1} \backslash\{0\}$ has an inverse element in $\mathbb{R}^{n+1}$ given by $z^{-1}=\frac{\bar{z}}{\|z\|^{2}}$.

In order to present the calculations in a more compact form, the following notations will be used, where $\mathbf{m}=\left(m_{1}, \ldots, m_{n}\right) \in \mathbb{N}_{0}^{n}$ is an $n$-dimensional multi-index:

$$
\mathbf{x}^{\mathbf{m}}:=x_{1}^{m_{1}} \cdots x_{n}^{m_{n}}, \quad \mathbf{m} !:=m_{1} ! \cdots m_{n} !, \quad|\mathbf{m}|:=m_{1}+\cdots+m_{n} .
$$

By $\tau(i)$ we denote the multi-index $\left(m_{1}, \ldots, m_{n}\right)$ with $m_{j}=\delta_{i j}$ for $1 \leq j \leq n$.

2.2. Clifford analysis. One way to generalize complex function theory to higher dimensional hypercomplex spaces is offered by the Riemann approach which considers Clifford algebra valued functions defined in $\mathbb{R}^{n+1}$ that are annihilated by the generalized Cauchy-Riemann operator

$$
D:=\frac{\partial}{\partial x_{0}}+\sum_{i=1}^{n} e_{i} \frac{\partial}{\partial x_{i}}
$$

If $U \subset \mathbb{R}^{n+1}$ is an open set, then a real differentiable function $f: U \rightarrow \mathbf{C l}_{0 n}$ is called left (right) monogenic or Clifford holomorphic at a point $z \in U$ if $D f(z)=0$ (or $f D(z)=0$ ). Functions that are left monogenic in the whole space are also called left entire. The notion of left (right) monogenicity in $\mathbb{R}^{n+1}$ provides indeed a powerful generalization of the concept of complex analyticity to Clifford analysis. Many classical theorems from complex analysis could be generalized to higher dimensions by this approach. We refer, e.g., to [2]. One central tool is the generalized Cauchy integral formula. 
Let us denote by $A_{n+1}$ the $n$-dimensional surface "area" of the $(n+1)$ dimensional unit ball, and by $q_{\mathbf{0}}(z)=\frac{\bar{z}}{\|z\|^{n+1}}$ the Cauchy kernel function. Then every function $f$ that is left monogenic in a neighborhood of the closure $\overline{\mathcal{D}}$ of a domain $\mathcal{D}$ satisfies

$$
f(z)=\frac{1}{A_{n+1}} \int_{\partial \mathcal{D}} q_{\mathbf{0}}(z-w) d \sigma(w) f(w)
$$

where $d \sigma(w)$ is the paravector-valued outer normal surface measure, i.e.,

$$
d \sigma(w)=\sum_{j=0}^{n}(-1)^{j} e_{j} \widehat{d w_{j}}
$$

with $\widehat{d w_{j}}=d w_{0} \wedge \cdots \wedge d w_{i-1} \wedge d w_{i+1} \wedge \cdots \wedge d w_{n}$.

It is important to mention that the set of left (right) monogenic functions forms only a Clifford right (left) module for $n>1$. Another important difference to classical complex function theory is that the ordinary powers of the hypercomplex variable $z$ are not monogenic. In the Clifford analysis setting, the complex positive powers are substituted by the so-called Fueter polynomials defined by

$$
\mathcal{P}_{\mathbf{m}}(z)=\frac{1}{|\mathbf{m}| !} \sum_{\pi \in \operatorname{perm}(\mathbf{m})} z_{\pi\left(m_{1}\right)} z_{\pi\left(m_{2}\right)} \cdots z_{\pi\left(m_{n}\right)}
$$

where $\operatorname{perm}(\mathbf{m})$ denotes the set of all permutations of the sequence $\left(m_{1}, m_{2}, \ldots\right.$, $\left.m_{n}\right)$, and $z_{i}:=x_{i}-x_{0} e_{i}$ for $i=1, \ldots, n$ and $\mathcal{P}_{\mathbf{0}}(z):=1$. In this underlying paper we prefer to work with the slightly modified Fueter polynomials

$$
V_{\mathbf{m}}(z):=\mathbf{m} ! \mathcal{P}_{\mathbf{m}}(z)
$$

which turns out to be more convenient in our calculations.

The negative power functions are generalized by the Cauchy kernel function $q_{\mathbf{0}}(z)$ and their partial derivatives

$$
q_{\mathbf{m}}(z)=\frac{\partial^{m_{0}+m_{1}+\cdots+m_{n}}}{\partial x_{0}^{m_{0}} \partial x_{1}^{m_{1}} \cdots \partial x_{n}^{m_{n}}} q_{\mathbf{0}}(z)
$$

In view of the monogenicity, one can restrict to consider only multi-indices with $m_{0}=0$ for many applications. In Taylor- and Laurent expansion theorems, these basic functions then take the same role as the ordinary powers do in complex function theory. 


\section{Order of growth of monogenic functions in $\mathbb{R}^{n+1}$}

The starting point for the following investigation is that monogenic functions $f: \mathbb{R}^{n+1} \rightarrow \mathbf{C l}_{0 n}$ satisfy a maximum principle (cf. [2]). Therefore, the function

$$
M(r, f):=M(r):=\max _{\|z\|=r}\{\|f(z)\|\}, \quad r \geq 0
$$

is well-defined and strictly monotonic increasing whenever $f$ is non-constant. Furthermore, by a direct verification we observe that $M$ is a continuous function.

It is extremely well-known that a complex-analytic polynomial $p(z)=$ $\sum_{n=0}^{N} a_{n} z^{n}$ grows asymptotically like $\left|a_{N}\right||z|^{N}$. In particular, they exhibit the asymptotic $|p(z)| \leq(1+\varepsilon)\left|a_{N}\right| r^{N}$. The standard monogenic Clifford algebra valued polynomial functions satisfy very similarly.

Theorem 3.1 (Asymptotic growth of the monogenic polynomials).

Let $P(z)=\sum_{|\mathbf{m}|=0}^{N} V_{\mathbf{m}}(z) a_{\mathbf{m}}$ be a left monogenic polynomial. Then one can find to every $\varepsilon>0$ an $r_{0}>0$ such that for all $r=\|z\| \geq r_{0}$

$$
\|P(z)\| \leq\left(\frac{(n-1+N) !}{(n-1) ! N !}+\varepsilon\right)\left\|a_{\mathbf{N}}\right\| r^{N},
$$

where $\mathbf{N}$ is an index with length $N$ such that $\left\|a_{\mathbf{N}}\right\| \geq\left\|a_{\mathbf{m}}\right\|$ for all $|\mathbf{m}|=N$.

Proof. According to, e.g., [6] and [13] we know that

$$
\left\|\mathcal{P}_{\mathbf{m}}(z)\right\| \leq \frac{\|z\|^{|\mathbf{m}|}}{\mathbf{m} !}
$$

hence $\left\|V_{\mathbf{m}}(z)\right\| \leq\|z\|^{|\mathbf{m}|}$. This leads to

$$
\begin{aligned}
\|P(z)\| & \leq \sum_{|\mathbf{m}|=0}^{N}\|z\|^{|\mathbf{m}|}\left\|a_{\mathbf{m}}\right\| \\
& \leq\left\|a_{\mathbf{N}}\right\|\|z\|^{N}\left(\sum_{|\mathbf{m}|=N} 1+\sum_{|\mathbf{m}|=0}^{N-1}\|z\|^{|\mathbf{m}|-N} \frac{\left\|a_{\mathbf{m}}\right\|}{\left\|a_{\mathbf{N}}\right\|}\right) \\
& =\left\|a_{\mathbf{N}}\right\| r^{N}\left(\frac{(n-1+N) !}{(n-1) ! N !}+r_{N}(z)\right),
\end{aligned}
$$

where we set

$$
r_{N}(z):=\sum_{|\mathbf{m}|=0}^{N-1}\|z\|^{|\mathbf{m}|-N} \frac{\left\|a_{\mathbf{m}}\right\|}{\left\|a_{\mathbf{N}}\right\|} .
$$


For a sufficiently large $r_{0}>0$ we observe that $\left|r_{N}(z)\right|<\varepsilon$ for all $\|z\|>r_{0}$, and therefore we infer that for all $\|z\|>r_{0}$

$$
\begin{aligned}
\|P(z)\| & \leq\left(\frac{(n-1+N) !}{(n-1) ! N !}+\left|r_{N}(z)\right|\right)\left\|a_{\mathbf{N}}\right\| r^{N} \\
& \leq\left(\frac{(n-1+N) !}{(n-1) ! N !}+\varepsilon\right)\left\|a_{\mathbf{N}}\right\| r^{N} .
\end{aligned}
$$

As it is well known, the maximum principle enables one to immediately set up a generalization of Cauchy's inequality, see e.g. [2]. Using the following inequalities from [14]

$$
\left\|q_{\mathbf{1}}(z)\right\|_{\|z\|=r} \leq \frac{n(n+1) \cdots(n+|\mathbf{l}|-1)}{r^{|\mathbf{1}|+n}},
$$

which are proved to be the optimal upper bound estimate (cf. [3]), Cauchy's inequality can be formulated in the following optimal way:

Theorem 3.2. Assume that $R>0$. Let $B(0, R):=\left\{z \in \mathbb{R}^{n+1} \mid\|z\| \leq R\right\}$. Suppose that $g: B(0, R) \rightarrow \mathbf{C l}_{0 n}$ is left monogenic with the Taylor expansion $g(z):=\sum_{|\mathbf{l}|=0}^{+\infty} V_{\mathbf{l}}(z) a_{\mathbf{l}}$. Then for all $0<r<R$ and all indices $\mathbf{l} \in \mathbb{N}_{0}^{n}$

$$
\left\|a_{\mathbf{l}}\right\| \leq M(r) \frac{n(n+1) \cdots(n+|\mathbf{l}|-1)}{\mathbf{l} ! r^{|\mathbf{l}|}}
$$

This follows directly by applying the estimate (7) and the maximum principle on the well-known representation formula for the Taylor coefficients

$$
a_{\mathbf{l}}=\frac{1}{\mathbf{l} ! A_{n+1}} \int_{\partial B(0, r)} q_{\mathbf{l}}(y) d \sigma(y) g(y) \text {. }
$$

Applying these inequalities to (9), then one arrives immediately at the stated result. ¿From Cauchy's inequality the generalization of the classical Liouville theorem (cf. [4], [7]), stating that every left entire monogenic function that is bounded in $\mathbb{R}^{n+1}$ is a constant, can be deduced directly.

Cauchy's inequality permits to also deduce the following more general version of Liouville's theorem.

Theorem 3.3. Suppose that $g: \mathbb{R}^{n+1} \rightarrow \mathbf{C l}_{0 n}$ is left entire (i.e., left monogenic in the whole space $\left.\mathbb{R}^{n+1}\right)$. If there exists an index $\mathbf{s} \in \mathbb{N}_{0}^{n}$ with $|\mathbf{s}|>0$ satisfying

$$
\liminf _{r \rightarrow \infty} \frac{M(r)}{r^{|\mathbf{s}|}}=L<\infty
$$

then

$$
g(z)=\sum_{|\mathbf{l}|=0}^{|\mathbf{s}|} V_{\mathbf{l}}(z) a_{\mathbf{l}}
$$


Proof. For a sequence $\left(r_{i}\right)_{i}$ with $r_{i} \rightarrow \infty$ we infer by (10) that

$$
\frac{M\left(r_{i}\right)}{r_{i}^{|\mathbf{s}|}} \leq L+1 .
$$

Applying Cauchy's inequality on the Taylor coefficients $a_{\mathbf{l}}$ of the function $g(z)=$ $\sum_{\mid \mathbf{l}=0}^{\infty} V_{\mathbf{l}}(z) a_{\mathbf{l}}$ in combination with (12) leads to

$$
\left\|a_{\mathbf{l}}\right\| \leq \frac{1}{\mathbf{l} !} n(n+1) \cdots(n+|\mathbf{l}|-1)(L+1) r_{i}^{|\mathbf{s}|-|\mathbf{l}|},
$$

from which follows that $a_{\mathbf{l}}=0$ for all $\mathbf{l}$ with $|\mathbf{l}|>|\mathbf{s}|$.

Remark. We observe that the expression

$$
\frac{\log (M(r, f))}{\log (r)}
$$

remains bounded if and only if $f$ is a monogenic polynomial, i.e. a function that can be written in the form $f(z)=\sum_{\mid \mathbf{l}=0}^{+s} V_{\mathbf{l}}(z) a_{\mathbf{l}}$, where $s$ is a finite nonnegative integer. By (13) we thus have characterized monogenic polynomials by the property of asymptotic growth.

Next we proceed to introduce the notion of the order of growth of monogenic functions. To this end we first introduce the plus logarithm (cf. e.g. [10]).

Definition 3.4. Let $\alpha \geq 0$. Then the plus logarithm is said to be

$$
\log ^{+}(\alpha):=\max \{0, \log (\alpha)\}
$$

In the same way as in the planar case (see [10]) one also may introduce order of growth for the hypercomplex case (see also [1]).

Definition 3.5. Let $g: \mathbb{R}^{n+1} \rightarrow \mathbf{C l}_{0 n}$ be a left entire function. Then

$$
\rho(g):=\limsup _{r \rightarrow \infty} \frac{\log ^{+}\left(\log ^{+} M(r)\right)}{\log (r)}, \quad 0 \leq \rho \leq \infty,
$$

is called the order of growth of the function $g$. We further introduce

$$
\lambda(g):=\liminf _{r \rightarrow \infty} \frac{\log ^{+}\left(\log ^{+} M(r)\right)}{\log (r)}, \quad 0 \leq \lambda \leq \infty,
$$

as the inferior order of growth of $g$. If $\rho=\lambda$, then we say that $g$ is a function of regular growth. If $\rho>\lambda$ then $g$ is said to be of irregular growth. 
Let us start with discussing some particular examples which play a fundamental role in Clifford analysis. We begin by looking at the simplest case where $P(z)$ is an arbitrary left monogenic polynomial, i.e., there exist Clifford numbers $a_{\mathbf{n}} \in \mathbf{C l}_{0 n}$ and $N \in \mathbb{N}_{0}$ such that $P(z)=\sum_{|\mathbf{n}|=0}^{N} V_{\mathbf{n}}(z) a_{\mathbf{n}}$. From Theorem 3.1 we know that

$$
\|P(z)\| \leq\left(\frac{(n-1+N) !}{(n-1) ! N !}+\varepsilon\right)\left\|a_{\mathbf{N}}\right\| r^{N},
$$

where $\mathbf{N}$ is the index of length $N$ for which $\left\|a_{\mathbf{N}}\right\| \geq\left\|a_{\mathbf{m}}\right\|$ for all $|\mathbf{m}|=N$, with an arbitrarily small $\varepsilon>0$ for $r$ sufficiently large. Thus, it follows with $C(N):=\left(\frac{(n-1+N) !}{(n-1) ! N !}+\varepsilon\right)\left\|a_{\mathbf{N}}\right\|$ that

$$
\lim _{r \rightarrow \infty} \frac{\log ^{+}\left(\log ^{+}(M(r, P))\right.}{\log (r)} \leq \lim _{r \rightarrow \infty} \frac{\log ^{+}\left(\log ^{+}\left(C(N) r^{N}\right)\right.}{\log (r)}=0 .
$$

Thus, all monogenic polynomials satisfy $\rho(P)=\lambda(P)=0$, like in the complex case.

In the classical case, the exponential function has growth order equal to 1. We shall now see that the different monogenic generalizations considered in $[2,5,8]$ turn out to have the same growth behavior.

The monogenic plane wave function from [5]

$$
P(\mathbf{m}, z):=(1+i \mathbf{m}) e^{-x_{0}} e^{i\langle\mathbf{m}, \mathbf{x}\rangle},
$$

where $\mathbf{m}$ is an arbitrary fixed vector from the $(n-1)$-dimensional unit sphere $S^{n}:=\left\{\mathbf{x} \in \mathbb{R}^{n} \mid\|\mathbf{x}\|=1\right\}$, is left entire and satisfies $\max _{\|z\|=r}\|P(\mathbf{m}, z)\|=$ $\|1+i \mathbf{m}\| e^{r}$. Hence, for $r>1$ we readily obtain

$$
\lim _{r \rightarrow \infty} \frac{\log ^{+}\left(\log ^{+}(M(r, P(\mathbf{m}, z)))\right.}{\log (r)}=\lim _{r \rightarrow \infty} \frac{\log ^{+}\left(\log ^{+}(\|1+i \mathbf{m}\|)+r\right)}{\log r}=1,
$$

i.e., $\rho(P(\mathbf{m}, z))=\lambda(P(\mathbf{m}, z))=1$ for all $\mathbf{m} \in S^{n}$.

Also the previously introduced monogenic generalization from $[2$, p. 117]

$$
\begin{aligned}
g(z) & =\exp \left(x_{0}, x_{1}, \ldots, x_{n}\right) \\
& =e^{x_{1}+\cdots+x_{n}}\left(\cos \left(x_{0} \sqrt{n}\right)-\frac{1}{\sqrt{n}}\left(e_{1}+\cdots+e_{n}\right) \sin \left(x_{0} \sqrt{n}\right)\right)
\end{aligned}
$$

satisfies $\|g(z)\|=e^{x_{1}+\cdots+x_{n}} \leq e^{n r}$. On the other hand we know that there must be a positive real number $0<c \leq n$ with $\max _{\|z\|=r}\|g(z)\| \geq e^{c r}$. The constant $c$ needs to be positive, otherwise we would have $\max _{\|z\|=r} e^{x_{1}+\cdots+x_{n}}=1$ which would be wrong. Hence,

$$
\lim _{r \rightarrow \infty} \frac{\log ^{+}\left(\log ^{+}(M(r, g))\right)}{\log (r)}=\lim _{r \rightarrow \infty} \frac{\log ^{+}\left(\log ^{+}\left(e^{c r}\right)\right)}{\log (r)}=1,
$$


with $0<c \leq n$, so that we get again $\rho(g)=\lambda(g)=1$, analogously to the classical case of dealing with the complex analytic exponential function.

Also the very recently introduced $\mathbb{R}^{n+1}$-valued multiperiodic exponential function from [8] fits within this scheme. To leave it simple, let us consider this function in the four-dimensional quaternionic case, where it has the representation

$$
\operatorname{EXP}\left(x_{0}, x_{1}, x_{2}, x_{3}\right)=\operatorname{Exp}_{0}+e_{1} \operatorname{Exp}_{1}+e_{2} \operatorname{Exp}_{2}+e_{3} \operatorname{Exp}_{3},
$$

where

$$
\begin{aligned}
& \operatorname{Exp}_{0}\left(x_{0}, x_{1}, x_{2}, x_{3}\right) \\
& =e^{x_{0}}\left(\cos \left(\frac{x_{1}}{\sqrt{3}}\right) \cos \left(\frac{x_{2}}{\sqrt{3}}\right) \cos \left(\frac{x_{3}}{\sqrt{3}}\right)-\sin \left(\frac{x_{1}}{\sqrt{3}}\right) \sin \left(\frac{x_{2}}{\sqrt{3}}\right) \sin \left(\frac{x_{3}}{\sqrt{3}}\right)\right) \\
& \operatorname{Exp}_{1}\left(x_{0}, x_{1}, x_{2}, x_{3}\right) \\
& =e^{x_{0}} \frac{\sqrt{3}}{3}\left(\sin \left(\frac{x_{1}}{\sqrt{3}}\right) \cos \left(\frac{x_{2}}{\sqrt{3}}\right) \cos \left(\frac{x_{3}}{\sqrt{3}}\right)+\cos \left(\frac{x_{1}}{\sqrt{3}}\right) \sin \left(\frac{x_{2}}{\sqrt{3}}\right) \sin \left(\frac{x_{3}}{\sqrt{3}}\right)\right) \\
& \operatorname{Exp}_{2}\left(x_{0}, x_{1}, x_{2}, x_{3}\right) \\
& =e^{x_{0}} \frac{\sqrt{3}}{3}\left(\cos \left(\frac{x_{1}}{\sqrt{3}}\right) \sin \left(\frac{x_{2}}{\sqrt{3}}\right) \cos \left(\frac{x_{3}}{\sqrt{3}}\right)+\sin \left(\frac{x_{1}}{\sqrt{3}}\right) \cos \left(\frac{x_{2}}{\sqrt{3}}\right) \sin \left(\frac{x_{3}}{\sqrt{3}}\right)\right) \\
& \operatorname{Exp}_{3}\left(x_{0}, x_{1}, x_{2}, x_{3}\right) \\
& =e^{x_{0}} \frac{\sqrt{3}}{3}\left(\sin \left(\frac{x_{1}}{\sqrt{3}}\right) \sin \left(\frac{x_{2}}{\sqrt{3}}\right) \cos \left(\frac{x_{3}}{\sqrt{3}}\right)+\cos \left(\frac{x_{1}}{\sqrt{3}}\right) \cos \left(\frac{x_{2}}{\sqrt{3}}\right) \sin \left(\frac{x_{3}}{\sqrt{3}}\right)\right) .
\end{aligned}
$$

By a direct computation one arrives at

$$
\frac{\sqrt{3}}{3} e^{r} \leq \max _{\|z\|=r}\left\|\operatorname{EXP}\left(x_{0}, x_{1}, x_{2}, x_{3}\right)\right\| \leq e^{r},
$$

hence, once more we obtain,

$$
\begin{aligned}
1=\lim _{r \rightarrow \infty} \frac{\log \left(\log \left(\frac{\sqrt{3}}{3} e^{r}\right)\right)}{\log (r)} & \leq \lim _{r \rightarrow \infty} \frac{\log ^{+}\left(\log ^{+} M(r, \mathrm{EXP})\right)}{\log (r)} \\
& \leq \lim _{r \rightarrow \infty} \frac{\log \left(\log \left(e^{r}\right)\right)}{\log (r)}=1
\end{aligned}
$$

After the discussion of these particular examples, let us now return to the more general framework. As a consequence of Cauchy's integral formula we can establish

Theorem 3.6. Let $g$ be a left entire function in $\mathbb{R}^{n+1}$. By $g_{i}$ we denote the function $g_{i}:=\frac{\partial}{\partial x_{i}} g$ and $M_{i}(r):=\max _{\|z\|=r}\left\{\left\|g_{i}(z)\right\|\right\}$, where $r>0$ and $i \in$ $\{0, \ldots, n\}$. Then

$$
\rho(g)=\rho^{\prime}(g) \text { and } \lambda(g)=\lambda^{\prime}(g),
$$


where

$$
\rho^{\prime}(g):=\limsup _{r \rightarrow \infty} \frac{\log ^{+}\left(\log ^{+}\left(M^{\prime}(r)\right)\right)}{\log (r)}, \quad \lambda^{\prime}(g):=\liminf _{r \rightarrow \infty} \frac{\log ^{+}\left(\log ^{+}\left(M^{\prime}(r)\right)\right)}{\log (r)},
$$

for $M^{\prime}(r):=\max _{i=0,1, \ldots, n}\left\{M_{i}(r)\right\}$.

Proof. Let us consider an arbitrary rectifiable curve from the origin to $z$. Then $g(z)=g(0)+\int_{0}^{1} \sum_{i=0}^{n} x_{i} g_{i}(t z) d t$. For $z \in \mathbb{R}^{n+1}$ with $\|z\|=r$ we get

$$
\|g(z)\| \leq\|g(0)\|+r \sum_{i=0}^{n} M_{i}(r) \leq\|g(0)\|+r(n+1) M^{\prime}(r) .
$$

Therefore we have $M(r) \leq\|g(0)\|+r(n+1) M^{\prime}(r)$. Applying some properties of $\log ^{+}$we obtain

$$
\log ^{+}(M(r)) \leq \log ^{+}(\|g(0)\|)+\log ^{+}(r(n+1))+\log ^{+}\left(M^{\prime}(r)\right)+\log (2),
$$

which leads to $\rho(g) \leq \rho^{\prime}(g)$ and $\lambda(g) \leq \lambda^{\prime}(g)$. To show the inequality in the other direction, we apply on $g_{i}$ Cauchy's integral formula:

$$
g_{i}(z)=\frac{1}{A_{n+1}} \int_{\|\zeta-z\|=R-r} q_{\tau(i)}(\zeta-z) d \sigma(\zeta) g(\zeta) .
$$

Applying the estimate (7) on (19) we hence obtain

$$
\left\|g_{i}(z)\right\| \leq \frac{1}{A_{n+1}} \int_{\|\zeta-z\|=R-r} \frac{n}{(R-r)^{n+1}} M(R) d S
$$

from which we then may infer $M_{i}(r) \leq \frac{n}{(R-r)} M(R)$, in particular for $M^{\prime}(r):=$ $\max _{i=0,1, \ldots, n}\left\{M_{i}(r)\right\}$ we have

$$
M^{\prime}(r) \leq \frac{n}{(R-r)} M(R)
$$

Setting $R=2 r$ in (20) we get $M^{\prime}(r) \leq \frac{n}{r} M(2 r)$. Thus,

$$
\log ^{+} M^{\prime}(r) \leq \log ^{+} M(2 r)+\log ^{+}\left(\frac{n}{r}\right) .
$$

For what follows we may assume without loss of generality that $r>n$. Thus, $\log ^{+} M^{\prime}(r) \leq \log ^{+} M(2 r)$. Hence,

$$
\frac{\log ^{+}\left(\log ^{+} M^{\prime}(r)\right)}{\log (r)} \leq \frac{\log ^{+}\left(\log ^{+} M(2 r)\right)}{\log (r)}=\frac{\log ^{+}\left(\log ^{+} M(2 r)\right)}{\log (2 r)} \frac{\log (2 r)}{\log (r)} .
$$

Thus, we have

$$
\frac{\log ^{+}\left(\log ^{+} M^{\prime}(r)\right)}{\log (r)} \leq \frac{\log ^{+}\left(\log ^{+} M(2 r)\right)}{\log (2 r)}\left(\frac{\log 2}{\log (r)}+1\right)
$$

from which we can infer directly that $\rho(g) \geq \rho^{\prime}(g)$ and $\lambda(g) \geq \lambda^{\prime}(g)$. 
Remark. Computing the growth order $\rho(f)$ resp. $\lambda(f)$ of a monogenic function $f$, then we automatically know the growth order of the maximum of all its partial derivatives which is the same.

Notice that Cauchy's integral formula was the fundamental ingredient to establish this result. It is thus indeed indispensable to work in classes of functions that are in the kernel of a differential operator that satisfy a Cauchy type integral formula. The class of monogenic functions provides us with the canonical and easiest example being endowed with this property.

\section{Central indices of an entire monogenic function in $\mathbb{R}^{n+1}$}

Take a left entire function $g(z)=\sum_{|\mathbf{l}|=0}^{+\infty} V_{\mathbf{l}}(z) a_{\mathbf{l}}$. If $g$ is transcendental, i.e., infinitely many $a_{\mathbf{l}} \neq 0$, then $\lim _{|\mathbf{1}| \rightarrow \infty}\left\|a_{\mathbf{l}}\right\| r^{|\mathbf{1}|}=0$ if one assumes $r$ to be fixed. Thus, the following expression is well-defined.

Definition 4.1. Let $g: \mathbb{R}^{n+1} \rightarrow \mathbf{C l}_{0 n}$ be a left entire function and let $r>0$ be a fixed real. Then the associated maximum term is said to be

$$
\mu(r):=\mu(r, g):=\max _{|\mathbf{1}| \geq 0}\left\{\left\|a_{\mathbf{1}}\right\| r^{|\mathbf{1}|}\right\}
$$

We further define central indices.

Definition 4.2. Let $g(z)=\sum_{|\mathbf{l}|=p}^{+\infty} V_{\mathbf{l}}(z) a_{\mathbf{l}}$ be a left entire function. For $r>0$ the index (or the indices) $\mathbf{m}$ with maximal length $|\mathbf{m}|$ with $\mu(r)=\left\|a_{\mathbf{m}}\right\| r^{|\mathbf{m}|}$ is (are) called central index (indices) which shall be denoted by $\nu(r)=\nu(r, g)=$ m. By $\nu(0)$ we denote the indices $\mathbf{l}$ which satisfy $|\mathbf{l}|=p$.

In a similar way we also can introduce these notions for monogenic polynomials. For a monogenic polynomial $p(z)=\sum_{|\mathbf{l}|=0}^{|\mathbf{N}|} V_{\mathbf{l}}(z) a_{\mathbf{l}}$, we observe that $\mu(r)=\left\|a_{\mathbf{N}}\right\| r^{|\mathbf{N}|}$ (where $\mathbf{N}$ is (are) the index (indices) of length $N$ satisfying $\left\|a_{\mathbf{N}}\right\| \geq\left\|a_{\mathbf{m}}\right\|$ for all $\left.|\mathbf{m}|=N\right)$ and $\nu(r)=\mathbf{N}$, provided $r$ is sufficiently large.

The case dealing with transcendental functions is more complicated. We show

Theorem 4.3. Assume that $g: \mathbb{R}^{n+1} \rightarrow \mathbf{C l}_{0 n}$ is a left entire transcendental function. Then

1. $\mu(r)$ increases for $r \leq r_{0}$ strictly monotonic and $\lim _{r \rightarrow \infty} \mu(r)=\infty$;

2. $|\nu(r)|$ increases monotonic and $\lim _{r \rightarrow \infty}|\nu(r)|=\infty$. Furthermore, $|\nu(r)|$ is stepwise constant. 
Proof. Ad 1. Since $g$ is not a constant function, we can infer that there exists an $r_{0}>0$ such that $|\nu(r)| \geq 1$ for $r \geq r_{0}$. From the definition we can further conclude that for $R>r \geq r_{0}$

$$
\mu(r)=\left\|a_{\nu(r)}\right\| r^{|\nu(r)|}<\left\|a_{\nu(r)}\right\| R^{|\nu(r)|} \leq\left\|a_{\nu(R)}\right\| R^{|\nu(R)|}=\mu(R),
$$

thus, $\mu(r)$ is strictly monotonic increasing.

Now we consider

$$
\begin{aligned}
\liminf _{r \rightarrow \infty} \frac{\log ^{+}(\mu(r))}{\log (r)} & \geq \liminf _{r \rightarrow \infty} \frac{\log \left(\left\|a_{\mathbf{l}}\right\| r^{|\mathbf{l}|}\right)}{\log (r)} \\
& =\liminf _{r \rightarrow \infty} \frac{\log \left\|a_{\mathbf{l}}\right\|+|\mathbf{l}| \log (r)}{\log (r)} \\
& =\liminf _{r \rightarrow \infty}\left(\frac{\log \left\|a_{\mathbf{l}}\right\|}{\log (r)}+|\mathbf{l}|\right)=|\mathbf{l}| \quad \forall \mathbf{l} \in \mathbb{N}_{0}^{n} .
\end{aligned}
$$

Since $g$ is a transcendental function, i.e., infinitely many $a_{1} \neq 0$, it follows that

$$
\liminf _{r \rightarrow \infty} \frac{\log ^{+}(\mu(r))}{\log (r)}=\infty
$$

i.e., $\mu(r) \rightarrow \infty$ for $r \rightarrow \infty$.

Ad 2. For $r<R$ we have the two estimates

$$
\begin{aligned}
\left\|a_{\nu(R)}\right\| R^{|\nu(R)|} & \geq\left\|a_{\nu(r)}\right\| R^{|\nu(r)|} \\
\left\|a_{\nu(r)}\right\| r^{|\nu(r)|} & \geq\left\|a_{\nu(R)}\right\| r^{|\nu(R)|}
\end{aligned}
$$

from which we infer that

$$
\left(\frac{R}{r}\right)^{|\nu(R)|} \geq\left(\frac{R}{r}\right)^{|\nu(r)|} .
$$

Thus, $|\nu(r)|$ is monotonic increasing.

From $\lim _{|\mathbf{1}| \rightarrow \infty} a_{\mathbf{l}}=0$ we infer that there is a positive constant $C$ such that $\mu(r)=\left\|a_{\nu(r)}\right\| r^{|\nu(r)|} \leq C r^{|\nu(r)|}$ from which

$$
\frac{\log ^{+}(\mu(r))}{\log (r)} \leq \frac{\log (C)}{\log (r)}+|\nu(r)|
$$

follows. From

$$
\lim _{r \rightarrow \infty} \frac{\log ^{+}(\mu(r))}{\log (r)}=\infty
$$

we conclude further that $\lim _{r \rightarrow \infty}|\nu(r)|=\infty$. Since $\nu(r) \in \mathbb{N}_{0}^{n}$ and since $|\nu(r)|$ tends monotonic to infinity, $|\nu(r)|$ has to be stepwise constant having at most a countable number of discontinuities. 


\section{Generalizations of some theorems from Valiron to Clifford analysis}

In this section we generalize some classical theorems from Valiron to Clifford analysis.

Theorem 5.1. Suppose that $g: \mathbb{R}^{n+1} \rightarrow \mathbf{C l}_{0 n}$ is a left entire transcendental function with the property that its first Taylor coefficient $a_{\mathbf{0}} \neq 0$. Then

$$
\log ^{+}(\mu(r))-\log \left\|a_{\mathbf{0}}\right\|=\int_{0}^{r} \frac{|\nu(t)|}{t} d t
$$

Proof. From the hypothesis we know that $g(z)=\sum_{|\mathbf{l}|=0}^{+\infty} V_{\mathbf{l}}(z) a_{\mathbf{l}}$ where infinitely many $a_{\mathbf{1}} \neq 0$, among them the first coefficient $a_{\mathbf{0}}=g(0)$.

First we assume without loss of generality that $g(0)=1$. If $0=t_{0}<t_{1}<$ $t_{2}<\ldots$ are the discontinuities of $|\nu(r)|$, then we can infer for $t_{j}<t<t_{j+1}$ that $\mu(t)=\left\|a_{\mathbf{l}}\right\| t^{|\mathbf{1}|}$ with a fixed $\mathbf{l} \equiv \nu(t)$. Furthermore, $\mu^{\prime}(t)=|\mathbf{l}|\left\|a_{\mathbf{l}}\right\| t^{|\mathbf{1}|-1}=$ $\frac{|\nu(t)|}{t} \mu(t)$. Thus, in an interval $[0, r]$ it holds excepted at a finite number of points

$$
\frac{d}{d t}\{\log (\mu(t))\}=\frac{\mu^{\prime}(t)}{\mu(t)}=\frac{|\nu(t)|}{t} .
$$

Since $\mu(t)$ is a continuous function, which can be proved in analogy as $[10$, Satz 4.2(b)], relying on Theorem 4.3, we can apply the main theorem of differential and integral calculus:

$$
\log (\mu(r))=\log (\mu(r))-\log (\mu(0))=\int_{0}^{r} \frac{d}{d t}\{\log (\mu(t))\} d t=\int_{0}^{r} \frac{|\nu(t)|}{t} d t .
$$

From Cauchy's inequality for monogenic functions (Theorem 3.2) we obtain immediately the estimate

$$
\mu(r) \leq M(r) \frac{n(n+1) \cdots(n+|\nu(r)|-1)}{\nu(r) !},
$$

where $\nu(r)$ is one central index.

Now we want to prove an estimate in the opposite direction. The following theorem provides a generalization of one of the classical Valiron theorems to Clifford analysis:

Theorem 5.2. If $g: \mathbb{R}^{n+1} \rightarrow \mathbf{C l}_{0 n}$ is a left entire function, then for all $0<r<R$

$$
M(r) \leq \mu(r)\left[|\nu(R)|(1+|\nu(R)|)^{n-1}+\frac{R}{R-r}\right] .
$$


Proof. The function $g$ is left entire, thus it can be represented by $g(z)=$ $\sum_{|\mathbf{l}|=0}^{+\infty} V_{\mathbf{l}}(z) a_{\mathbf{l}}$ where infinitely many $a_{\mathbf{l}} \neq 0$, since $g$ is transcendental. From the maximum modulus theorem for monogenic functions we infer that for $0<r<R$

$$
\begin{aligned}
M(r) & \leq \sum_{|\mathbf{1}|=0}^{+\infty}\left\|a_{\mathbf{l}}\right\| r^{|\mathbf{1}|} \\
& =\sum_{|\mathbf{1}|=0}^{|\nu(R)|-1}\left\|a_{\mathbf{l}}\right\| r^{|\mathbf{1}|}+\sum_{|\mathbf{1}|=|\nu(R)|}^{+\infty}\left\|a_{\mathbf{l}}\right\| r^{|\mathbf{1}|} \\
& \leq \sum_{|\mathbf{|}|=0}^{|\nu(R)|-1} \mu(r)+\sum_{|\mathbf{1}|=|\nu(R)|}^{+\infty}\left\|a_{\mathbf{l}}\right\| r^{|\mathbf{1}|} .
\end{aligned}
$$

In view of

$$
\begin{aligned}
\sum_{|\mathbf{1}|=0}^{|\nu(R)|-1} 1 & =\sum_{|\mathbf{1}|=0} 1+\sum_{|\mathbf{1}|=1} 1+\cdots+\sum_{|\mathbf{1}|=|\nu(R)|-1} 1 \\
& =1+\frac{((n-1)+1) !}{(n-1) ! 1 !}+\cdots+\frac{[(n-1)+(\mid \nu(R)-1)] !}{(n-1) !(|\nu(R)|-1) !} \\
& \leq|\nu(R)|\left[\frac{[(n-1)+|\nu(R)|-1] !}{(n-1) !(|\nu(R)|-1) !}\right]
\end{aligned}
$$

where we use that for all $n \geq 1$ the inequality

$$
\frac{(n-1+k) !}{(n-1) ! k !} \leq \frac{(n-1+(k+1)) !}{(n-1) !(k+1) !}
$$

holds, which itself can be verified by a straightforward induction over $k$. Further,

$$
\begin{aligned}
& |\nu(R)|\left[\frac{[(n-1)+|\nu(R)|-1] !}{(n-1) !(|\nu(R)|-1) !}\right] \\
& =|\nu(R)|\left[\frac{(|\nu(R)|+n-2)(|\nu(R)|+n-3) \cdots(|\nu(R)|+1)|\nu(R)|}{(n-1) !}\right] \\
& =|\nu(R)|\left[\frac{|\nu(R)|+n-2}{n-1} \cdot \frac{|\nu(R)|+n-3}{n-2} \cdots \frac{|\nu(R)|+1}{2} \cdot \frac{|\nu(R)|}{1}\right] \\
& \leq|\nu(R)|\left[(\underbrace{1+\frac{|\nu(R)|}{n-1}}_{\leq 1+|\nu(R)|})(\underbrace{1+\frac{|\nu(R)|}{n-2}}_{\leq 1+|\nu(R)|}) \cdots(\underbrace{1+\frac{|\nu(R)|}{1}}_{=1+|\nu(R)|})\right] \\
& \leq|\nu(R)|\left[(1+|\nu(R)|)^{n-1}\right] .
\end{aligned}
$$


Inserting these results into (25) leads to

$$
\begin{aligned}
& M(r) \leq \mu(r)|\nu(R)|\left[(1+|\nu(R)|)^{n-1}\right]+\sum_{|\mathbf{1}|=|\nu(R)|}^{+\infty}\left\|a_{\mathbf{l}}\right\| r^{|\mathbf{l}|} \frac{\left\|a_{\nu(r)}\right\| r^{|\nu(r)|} R^{|\mathbf{1}+\nu(R)|}}{\left\|a_{\nu(R)}\right\| r^{|\nu(R)|} R^{|\mathbf{l}+\nu(R)|}} \\
& =\mu(r)|\nu(R)|\left[(1+|\nu(R)|)^{n-1}\right]+\mu(r) \sum_{|\mathbf{l}|=|\nu(R)|}^{+\infty} \frac{\left\|a_{\mathbf{1}}\right\| R^{|\mathbf{l}|} R^{|\nu(R)|} r^{|\mathbf{1}|}}{\left\|a_{\nu(R)}\right\| R^{|\nu(R)|} R^{|\mathbf{1}|} r^{|\nu(R)|}} \\
& \leq \mu(r)|\nu(R)|\left[(1+|\nu(R)|)^{n-1}\right]+\mu(r) \sum_{|\mathbf{1}|=|\nu(R)|}^{+\infty}\left(\frac{r}{R}\right)^{|\mathbf{1}|-|\nu(R)|} \\
& =\mu(r)\left[|\nu(R)|\left[(1+|\nu(R)|)^{n-1}\right]+\frac{R}{R-r}\right] \text {. }
\end{aligned}
$$

In complex analysis G. Valiron has proved that an entire complex-analytic function $g$ of finite order shows the asymptotic behavior $\log (M(r)) \sim \log \left(M^{\prime}(r)\right)$, where $M^{\prime}$ is the maximum modulus of the derivative. The classical proof is based on the fact that one has the relation $\mu(r) \leq M(r)$ for complex-analytic function. In the framework of working with Clifford algebra valued monogenic Taylor series built with the Fueter polynomials, we have been so far only able to establish an estimate of the form $\mu(r) \leq \frac{n(n+1) \cdots(n+|\nu(r)|-1)}{\nu(r) !} M(r)$ for a central in$\operatorname{dex} \nu(r)$ as a consequence of Cauchy's inequalities. Notice that the estimate (7) has been proven to be sharp. Adapting the classical methods based on Cauchy's inequality to the higher dimensional case provides us only with a slightly weaker result in the Clifford analysis setting. First we show

Proposition 5.3. For a left entire function $g: \mathbb{R}^{n+1} \rightarrow \mathbf{C l}_{0 n}$ of order $\rho$ and inferior order $\lambda$ set

$$
\begin{array}{lll}
\rho_{1}:=\limsup _{r \rightarrow \infty} \frac{\log ^{+} \log ^{+} \mu(r)}{\log (r)}, & \rho_{2}:=\limsup _{r \rightarrow \infty} \frac{\log ^{+}|\nu(r)|}{\log (r)} \\
\lambda_{1}:=\liminf _{r \rightarrow \infty} \frac{\log ^{+} \log ^{+} \mu(r)}{\log (r)}, & \lambda_{2}:=\liminf _{r \rightarrow \infty} \frac{\log ^{+}|\nu(r)|}{\log (r)} .
\end{array}
$$

Then $\rho \leq \rho_{1}=\rho_{2}$ and $\lambda \leq \lambda_{1}=\lambda_{2}$.

Proof. The proof that $\rho_{1}=\rho_{2}$ and $\lambda_{1}=\lambda_{2}$ can be done in complete analogy to the complex case presented in [10, Satz 4.5]. Hence we omit this part.

Let us now show that $\rho \leq \rho_{1}$. Without loss of generality we may restrict to consider the case where $\rho_{1}<\infty$, since the assertion is trivial in the remaining case where $\rho_{1}=\infty$. Inserting in particular $r=\frac{R}{2}$ into Theorem 5.2, leads to

$$
M(r) \leq \mu(r)\left[|\nu(2 r)|[1+|\nu(2 r)|]^{n-1}+2\right] .
$$


In view of

$$
\frac{\log |\nu(r)|}{\log (r)} \leq \rho_{2}+\varepsilon, \quad \varepsilon>0,
$$

which equivalently reads $|\nu(r)| \leq e^{\left(\rho_{2}+\varepsilon\right) \log (r)}=r^{\rho_{2}+\varepsilon}$, we thus may conclude that for a sufficiently large $r$ there is an $\varepsilon_{1}>0$ and a $\delta>0$ such that

$$
\begin{aligned}
M(r) & \leq \mu(r)\left(|\nu(2 r)|^{n}\left(1+\varepsilon_{1}\right)\right) \\
& \leq \mu(r)\left((2 r)^{n\left(\rho_{2}+\varepsilon\right)}\left(1+\varepsilon_{1}\right)\right) \\
& \leq \mu(r)(2 r)^{n \rho_{2}+n \varepsilon}(2 r)^{\delta} .
\end{aligned}
$$

Hence with $\varepsilon_{2}:=n \varepsilon+\delta$ we thus have

$$
M(r) \leq \mu(r)(2 r)^{n \rho_{2}+\varepsilon_{2}} .
$$

We thus obtain

$$
\begin{aligned}
\frac{\log ^{+}\left(\log ^{+} M(r)\right)}{\log (r)} & \leq \frac{\log ^{+} \log ^{+}\left[\mu(r)(2 r)^{n \rho_{2}+\varepsilon_{2}}\right]}{\log (r)} \\
& =\frac{\log ^{+}\left[\log ^{+} \mu(r)+\log ^{+}(2 r)^{n \rho_{2}+\varepsilon_{2}}\right]}{\log (r)} \\
& \leq \frac{\log ^{+}\left(\log ^{+}(\mu(r))\right)+\log ^{+}\left(\log ^{+}\left((2 r)^{n \rho_{2}+\varepsilon_{2}}\right)\right)+\log (2)}{\log (r)} \\
& \leq \frac{\log ^{+}\left(\log ^{+} \mu(r)\right)+\log ^{+}\left(\left(n \rho_{2}+\varepsilon_{2}\right) \log ^{+}(2 r)\right)+\log (2)}{\log (r)} .
\end{aligned}
$$

We thus obtain that

$$
\limsup _{r \rightarrow \infty} \frac{\log ^{+}\left(\log ^{+} M(r)\right)}{\log (r)} \leq \limsup _{r \rightarrow \infty} \frac{\log ^{+}\left(\log ^{+} \mu(r)\right)}{\log (r)}=\rho_{1} .
$$

Let us now show that $\lambda \leq \lambda_{2}\left(=\lambda_{1}\right)$. If $\lambda_{2}=\infty$, then the assertion is trivial. Let us thus assume without loss of generality that $\lambda_{2}<\infty$. Then there exists a sufficiently large $R$ such that $\frac{\log ^{+}|\nu(R)|}{\log R} \leq \lambda_{2}+\varepsilon$, which equivalently reads

$$
|\nu(R)| \leq R^{\lambda_{2}+\varepsilon}
$$

Since the Taylor series converges we hence may conclude that we have for sufficiently large $R$

$$
\mu(R)=\| a_{\nu(R)}|| R^{|\nu(R)|} \leq R^{|\nu(R)|} \leq R^{R^{\lambda_{2}+\varepsilon}} .
$$

Inserting $r=\frac{R}{2}$ into Theorem 5.2 and applying (30) and (31) thus leads to

$$
M(r) \leq R^{R^{\lambda_{2}+\varepsilon}}\left[R^{n \lambda_{2}+n \varepsilon}\left(1+\varepsilon^{\prime}\right)\right] \leq R^{R^{\lambda_{2}+\varepsilon+\delta}} R^{n \lambda_{2}+n \varepsilon+\delta} \leq R^{R^{\lambda_{2}+\varepsilon+\delta^{\prime}}} \leq r^{r^{\lambda_{2}+\varepsilon_{1}}}
$$


with some appropriate $\varepsilon^{\prime}, \varepsilon^{*}, \varepsilon_{1}, \delta, \delta^{\prime}>0$. We finally obtain

$$
\liminf _{r \rightarrow \infty} \frac{\log ^{+}\left(\log ^{+} M(r)\right)}{\log (r)} \leq \liminf _{r \rightarrow \infty} \frac{\log ^{+}\left(r^{\lambda_{2}+\varepsilon_{1}} \log r\right)}{\log (r)}=\lambda_{2}=\lambda_{1} .
$$

Remark. In the two-dimensional complex case where we have $\mu(r) \leq M(r)$ these methods allow one to establish the stronger result $\rho=\rho_{1}=\rho_{2}$ and $\lambda=\lambda_{1}=\lambda_{2}$, as shown for instance in [10, Satz 4.5].

With this proposition we may now establish the following theorem which provides us with a weaker analogy of Valiron's asymptotic result on the growth of the logarithm of the derivative of a given analytic function.

Theorem 5.4. If $g: \mathbb{R}^{n+1} \rightarrow \mathbf{C l}_{0 n}$ is left entire with $\rho_{2}(g)<\infty$, then

$$
\limsup _{r \rightarrow \infty} \frac{\log M_{i}(r)}{\log \mu(r)} \leq 1
$$

where $M_{i}(r):=\max _{\|z\|=r}\left\{\left\|\frac{\partial}{\partial x_{i}} g(z)\right\|\right\}$ for $i=1, \ldots, n$.

Proof. Since $g$ is left entire, we have $g(z)=\sum_{|\mathbf{m}|=0}^{+\infty} V_{\mathbf{m}}(z) a_{\mathbf{m}}$ in the whole space $\mathbb{R}^{n+1}$, hence so is

$$
\begin{aligned}
g_{i}(z):=\frac{\partial}{\partial x_{i}} g(z) & =\sum_{|\mathbf{m}|=0}^{+\infty} \frac{\partial}{\partial x_{i}} V_{\mathbf{m}}(z) a_{\mathbf{m}} \\
& =\sum_{|\mathbf{m}|=1}^{+\infty} \frac{\mathbf{m} !}{(\mathbf{m}-\tau(i)) !} V_{\mathbf{m}-\tau(i)}(z) a_{\mathbf{m}} .
\end{aligned}
$$

Therefore, we obtain for $\|z\|=r$

$$
\left\|g_{i}(z)\right\| \leq \sum_{|\mathbf{m}|=1}^{+\infty} \frac{\mathbf{m} !}{(\mathbf{m}-\tau(i)) !}\left\|a_{\mathbf{m}}\right\| r^{|\mathbf{m}-\tau(i)|} .
$$

In what follows let us denote by $\mu_{i}$ the maximum term of $g_{i}$ and similarly by $\nu_{i}$ the central indices of $g_{i}$. If $\mu(r)=\left\|a_{\mathbf{m}}\right\| r^{|\mathbf{m}|}$, then $\mathbf{m}=\nu(r)$ and hence we can conclude that

$$
\mu_{i}(r) \leq \| a_{\nu(r)}|| r^{|\nu(r)|-1}|\nu(r)|=\mu(r) \frac{1}{r}|\nu(r)| .
$$

In view of $|\nu(r)|=\left|\nu_{i}(r)\right|+1$ we thus may infer that

$$
\limsup _{r \rightarrow \infty} \frac{\log ^{+}\left(\left|\nu_{i}(r)\right|+1\right)}{\log (r)}=\limsup _{r \rightarrow \infty} \frac{\log ^{+}|\nu(r)|}{\log (r)}=: \rho_{2} .
$$


Further to this we have for $\varepsilon>0$ that $\left|\nu_{i}(r)\right| \leq\left|\nu_{i}(r)\right|+1 \leq r^{\rho_{2}+\varepsilon}$. Applying Theorem 5.2 and the same arguments as in the proof of Proposition 5.3, for $\varepsilon_{1}>0$ we finally arrive at

$$
M_{i}(r) \leq \mu_{i}(r) r^{n \rho_{2}+\varepsilon_{1}} \stackrel{(34)}{\leq} \mu(r)|\nu(r)| r^{n \rho_{2}+\varepsilon_{1}-1} \leq \mu(r) r^{(n+1) \rho_{2}+\varepsilon_{1}+\varepsilon-1},
$$

since $|\nu(r)|<r^{\rho_{2}+\varepsilon}$. Finally, putting $\delta_{1}:=\varepsilon_{1}+\varepsilon$ this then leads to

$$
\begin{aligned}
\log M_{i}(r) & \leq \log \mu(r)+\log r^{(n+1) \rho_{2}+\delta_{1}-1} \\
& =\log \mu(r)+\left[(n+1) \rho_{2}+\delta_{1}-1\right] \log r,
\end{aligned}
$$

which permits us to conclude that

$$
\limsup _{r \rightarrow \infty} \frac{\log M_{i}(r)}{\log \mu(r)} \leq \limsup _{r \rightarrow \infty}\left(1+\left((n+1) \rho_{2}+\delta_{1}-1\right) \frac{\log r}{\log \mu(r)}\right)=1 .
$$

\section{Action of iterated Euler operators}

In this section we want to establish a relation between the asymptotic behavior of the maximum term of a monogenic function and that of their iterated radial derivatives. To proceed in this direction we first establish some preparatory propositions.

In what follows in this section we will sometimes write for convenience $\nu:=\nu(r)=\nu(r, g)$ for a left entire function $g: \mathbb{R}^{n+1} \rightarrow \mathbf{C l}_{0 n}$ in cases when no ambiguity can occur.

Proposition 6.1. Let $g$ be a transcendental left entire monogenic function. Then

$$
M(r, g) \leq \mu(r) L(r)
$$

where

$$
L(r):=\frac{((n-1)+|\nu|) !}{(n-1) !|\nu| !}+\sum_{|\mathbf{1}|=0}^{|\nu|-1} \frac{\left\|a_{\mathbf{1}}\right\|}{\left\|a_{\nu}\right\|} r^{|1|-|\nu|}+\sum_{|\mathbf{l}|=|\nu|+1}^{+\infty} \frac{\left\|a_{\mathbf{l}}\right\|}{\left\|a_{\nu}\right\|} r^{|1|-|\nu|} .
$$

Proof. Since $g$ is a transcendental left entire monogenic function it has a Taylor expansion (with infinitely many non-vanishing terms) of the form $g(z)=$ $\sum_{|\mathbf{l}|=0}^{+\infty} V_{\mathbf{l}}(z) a_{\mathbf{l}}$. We have $\|g(z)\| \leq \sum_{|\mathbf{l}|=0}^{+\infty}\left\|V_{\mathbf{l}}(z)\right\|\left\|a_{\mathbf{l}}\right\| \leq \sum_{|\mathbf{l}|=0}^{+\infty} r^{|\mathbf{l}|}\left\|a_{\mathbf{l}}\right\|$ for $\|z\|=r$. Since $g$ is transcendental, there exists $\mathbf{k} \in \mathbb{N}_{0}^{n}$ such that $a_{\mathbf{k}} \neq 0$. Then

$$
\|g(z)\| \leq r^{|\mathbf{k}|}\left\|a_{\mathbf{k}}\right\|\left[\sum_{|\mathbf{1}|=|\mathbf{k}|} \frac{\left\|a_{\mathbf{l}}\right\|}{\left\|a_{\mathbf{k}}\right\|}+\sum_{|\mathbf{1}|=0}^{|\mathbf{k}|-1} \frac{\left\|a_{\mathbf{1}}\right\|}{\left\|a_{\mathbf{k}}\right\|} r^{|\mathbf{1}|-|\mathbf{k}|}+\sum_{|\mathbf{1}|=|\mathbf{k}|+1}^{+\infty} \frac{\left\|a_{\mathbf{l}}\right\|}{\left\|a_{\mathbf{k}}\right\|} r^{|\mathbf{1}|-|\mathbf{k}|}\right] .
$$

Taking in particular $\mathbf{k}=\nu(r)$ and involve $\mu(r)=\left\|a_{\mathbf{k}}\right\| r^{|\mathbf{k}|}$, then one arrives at the stated result. 
In order to proceed we need the following

Proposition 6.2. Let $\left(P_{k}\right)_{k \in \mathbb{N}}$ be a sequence of real positives satisfying $1<$ $P_{1}<P_{2}<\ldots$ and $\lim _{k \rightarrow \infty} P_{k}=P<\infty$. Then there exists a real $r>0$ such that

$$
\begin{array}{ll}
\frac{\left\|a_{\nu-\mathbf{j}}\right\| r^{|\nu|-|\mathbf{j}|}}{\left\|a_{\nu}\right\| r^{|\nu|}} \leq \frac{\prod_{i=|\nu|-|\mathbf{j}|+1}^{|\nu|} P_{i}}{P_{|\nu|}^{|\mathbf{j}|}}, \quad|\mathbf{j}|=1, \ldots,|\nu| \\
\frac{\left\|a_{\nu+\mathbf{j}}\right\| r^{|\nu+\mathbf{j}|}}{\left\|a_{\nu}\right\| r^{|\nu|}}<\frac{P_{|\nu|}^{|\mathbf{j}|}}{\prod_{i=|\nu|+1}^{|\nu+\mathbf{j}|} P_{i}}, \quad|\mathbf{j}|=1,2,3, \ldots
\end{array}
$$

The proof can be done in complete analogy to the classical proof of from [10, Hilfsatz 21.], p. 189, simply by replacing the auxiliary function $H(z)$ defined on p. 190, equation (21.9), by

$$
H(z)=\sum_{|\mathbf{n}|=0}^{+\infty} V_{\mathbf{n}}(z) a_{\mathbf{n}}\left(\prod_{i=1}^{|\mathbf{n}|} P_{i}\right) .
$$

The rest of the proof can then be carried over directly.

Further to this we require

Proposition 6.3. For a given $\varepsilon>0$ we have

$$
\begin{array}{ll}
L^{*}(r)<|\nu(r)|^{\frac{1}{2}+\varepsilon}, & r \notin F \\
|\nu(r)|<(\log \mu(r))^{1+\varepsilon}, & r \notin F,
\end{array}
$$

where $L^{*}(r):=L(r)-\left(\frac{((n-1)+|\nu|) !}{(n-1) !|\nu| !}-1\right)$ and $F$ denotes a set of finite logarithmic measure.

and also

Proposition 6.4. For $\varepsilon>0, k^{\prime}, l \in \mathbb{N}, k \in \mathbb{N}_{0}$ and $\mathbf{m} \in \mathbb{N}_{0}^{n}$ one has

$$
\begin{array}{ll}
\sum_{[\mathbf{j}]=-|\nu|}^{+\infty}\left(\frac{|\mathbf{j}|}{|\nu|}\right)^{k^{\prime}}\left\|a_{\nu+\mathbf{j}}\right\| r^{[\nu+\mathbf{j}]}<\mu(r)|\nu(r)|^{\frac{1-k^{\prime}}{2}+\varepsilon}, & r \notin F \\
\sum_{[\mathbf{j}]=[\mathbf{m}-\nu]}^{+\infty} \frac{|[\mathbf{j}]|^{k}}{|\nu|^{k+l}}\left\|a_{\nu+\mathbf{j}}\right\| r^{[\mathbf{j}+\nu]}<\mu(r)|\nu(r)|^{-\frac{1}{2}+\varepsilon}, & r \notin F .
\end{array}
$$


Here, the notation $[\mathbf{j}]$ means $[\mathbf{j}]=\sum_{i=1}^{n} j_{i}$. Notice that the first indices $\mathbf{j}$ appearing in this sum are elements from $-\mathbb{N}_{0}^{n}$. The expression $[\mathbf{j}]$ coincides with the previously introduced length of an index for all $\mathbf{j} \in \mathbb{N}_{0}^{n}$.

Using Proposition 6.2 one can prove these propositions in an analogous way as their counterparts from the complex analysis setting presented in Satz 21.1, Satz 21.2, and in Hilfssatz 21.2 and Hilfssatz 21.3 from [10], respectively. These propositions enable us to establish the main theorem of this section.

Theorem 6.5. Let $g: \mathbb{R}^{n+1} \rightarrow \mathbf{C l}_{0 n}$ be a left entire function. Then for all $k \in \mathbb{N}$ holds asymptotically

$$
\left\|\frac{1}{|\nu(r)|^{k}}\left[E^{k}\right] g(z)-g(z)\right\| \leq C \mu(r)|\nu(r)|^{-\frac{1}{2}+\varepsilon}, \quad r \notin F,
$$

where $E:=\sum_{i=0}^{n} x_{i} \frac{\partial}{\partial x_{i}}$ is the Euler operator on $\mathbb{R}^{n+1}, C$ is a real positive constant and $F$ is a set of finite logarithmic measure.

Proof. To show this result, we start to consider

$$
\begin{aligned}
& \frac{1}{|\nu|^{k}}\left[\sum_{i=0}^{n} x_{i} \frac{\partial}{\partial x_{i}}\right]^{k} g(z)-g(z) \\
& =\frac{1}{|\nu|^{k}} \sum_{|\mathbf{m}|=1}^{+\infty}|\mathbf{m}|^{k} V_{\mathbf{m}}(z) a_{\mathbf{m}}-\sum_{|\mathbf{m}|=0}^{+\infty} V_{\mathbf{m}}(z) a_{\mathbf{m}} \\
& =-a_{\mathbf{0}}+\frac{1}{|\nu|^{k}}\left[\sum_{|\mathbf{m}|=1}^{+\infty}\left(|\mathbf{m}|^{k}-|\nu|^{k}\right) V_{\mathbf{m}}(z) a_{\mathbf{m}}\right] \\
& |\mathbf{m}|=\underline{\underline{\mathbf{j}}]}+|\nu|+a_{\mathbf{0}}+\frac{1}{|\nu|^{k}}\left[\sum_{[\mathbf{j}]=1-|\nu|}^{+\infty}([\mathbf{j}]+|\nu|)^{k}-|\nu|^{k}\right] V_{\mathbf{j}+\nu}(z) a_{\mathbf{j}+\nu} \\
& =-a_{\mathbf{0}}+\frac{1}{|\nu|^{k}} \sum_{[\mathbf{j}]=1-|\nu|}^{+\infty}\left(\sum_{s=0}^{k}[\mathbf{j}]^{s}|\nu|^{k-s}\left(\begin{array}{c}
k \\
s
\end{array}\right)-|\nu|^{k}\right) V_{\mathbf{j}+\nu}(z) a_{\mathbf{j}+\nu} \\
& =-a_{\mathbf{0}}+\sum_{[\mathbf{j}]=1-|\nu|}^{+\infty} \sum_{s=1}^{k}[\mathbf{j}]^{s}|\nu|^{-s}\left(\begin{array}{l}
k \\
s
\end{array}\right) V_{\mathbf{j}+\nu}(z) a_{\mathbf{j}+\nu} \\
& =-a_{\mathbf{0}}+\sum_{[\mathbf{j}]=1-|\nu|}^{+\infty} \sum_{s=1}^{k}|\nu|^{-s} \underbrace{P_{s}([\mathbf{j}])}_{:=[\mathbf{j}]^{s}\left(\begin{array}{l}
k \\
s
\end{array}\right)} V_{\mathbf{j}+\nu}(z) a_{\mathbf{j}+\nu} .
\end{aligned}
$$

Let us now estimate the expression

$$
S_{s}(z)=\sum_{[\mathbf{j}]=1-|\nu|}^{+\infty}|\nu|^{-s} P_{s}([\mathbf{j}]) V_{\mathbf{j}+\nu}(z) a_{\mathbf{j}+\nu}
$$


Taking $\|z\|=r>0$, we have

$$
\begin{aligned}
\left\|S_{s}(z)\right\| & \leq \sum_{[\mathbf{j}]=1-|\nu|}^{+\infty}|\nu|^{-s} P_{s}(|\mathbf{j}|)\left\|a_{\mathbf{j}+\nu}\right\|\left\|V_{\mathbf{j}+\nu}(z)\right\| \\
& \leq \sum_{[\mathbf{j}]=1-|\nu|}^{+\infty}|\nu|^{-s} P_{s}(|\mathbf{j}|)\left\|a_{\mathbf{j}+\nu}\right\| r^{[\mathbf{j}+\nu]} \\
& =\sum_{[\mathbf{j}]=1-|\nu|}^{+\infty}\left(\frac{|\mathbf{j}|}{|\nu|}\right)^{s} \frac{k !}{(k-s) ! s !}\left\|a_{\mathbf{j}+\nu}\right\| r^{[\mathbf{j}+\nu]} .
\end{aligned}
$$

We thus have

$$
\left\|S_{s}(z)\right\| \leq \frac{k !}{(k-s) ! s !} \sum_{[\mathbf{j}]=1-|\nu|}^{+\infty}\left(\frac{|\mathbf{j}|}{|\nu|}\right)^{s}\left\|a_{\mathbf{j}+\nu}\right\| r^{[\mathbf{j}+\nu]} .
$$

Applying Proposition 6.4 for the particular case $l=0$ and $|\mathbf{m}|=1$ to the previous line, we thus obtain

$$
\left\|S_{s}(z)\right\| \leq \frac{k !}{(k-s) ! s !} \mu(r)|\nu(r)|^{-\frac{1}{2}+\varepsilon}, \quad r \notin F .
$$

Summarizing, we thus obtain

$$
\begin{aligned}
\| \frac{1}{|\nu|^{k}}\left[\sum_{i=0}^{n} x_{i} \frac{\partial}{\partial x_{i}}\right]^{k} g(z) & -g(z) \| \\
& \leq\left\|a_{\mathbf{0}}\right\|+\sum_{s=1}^{k}\left\|S_{s}(z)\right\| \\
& \leq\left\|a_{\mathbf{0}}\right\|+\sum_{s=1}^{k} \frac{k !}{(k-s) ! s !} \mu(r)|\nu(r)|^{-\frac{1}{2}+\varepsilon} \\
& =\left\|a_{\mathbf{0}}\right\|+\left(2^{k}-1\right) \mu(r)|\nu(r)|^{-\frac{1}{2}+\varepsilon} \\
& \leq
\end{aligned}
$$

for $r \notin F$, where $C$ is a positive real constant.

As an application we may set up the following

Proposition 6.6. Let $0<\delta<\frac{1}{2}$ and $\|z\|$ be sufficiently large such that for $\|z\|=r$, the relation

$$
\|g(z)\|>\mu(r)|\nu(r)|^{-\frac{1}{2}+\delta}
$$


is satisfied, where $g$ is assumed to be left entire. Then for all $k \in \mathbb{N}$ holds asymptotically

$$
\frac{1}{|\nu(r)|^{k}}\left[E^{k}\right] g(z)-g(z)=o(1) g(z), \quad r \notin F
$$

where $F$ is again a set of finite logarithmic measure.

Proof. Let us now suppose that $\|z\|=r \notin F$. In view of condition (46) we have

$$
\begin{aligned}
\frac{1}{\|g(z)\|}\left\|\frac{1}{|\nu(r)|^{k}}\left[\sum_{i=0}^{n} x_{i} \frac{\partial}{\partial x_{i}}\right]^{k} g(z)-g(z)\right\| & \leq \frac{C}{\mu(r)}|\nu(r)|^{\frac{1}{2}-\delta} \mu(r)|\nu(r)|^{-\frac{1}{2}+\varepsilon} \\
& =C|\nu(r)|^{\varepsilon-\delta} \longrightarrow 0
\end{aligned}
$$

if we choose $\varepsilon$ sufficiently small (i.e., $\varepsilon<\delta$ ). In other words, we indeed have $\frac{1}{|\nu|^{k}}\left[E^{k}\right] g(z)-g(z)=o(1) g(z)$ under the given condition.

Remark. This statement provides us with a nice analogy in the context of Clifford analysis of the classical Satz 21.3 from [10] which states that entire complex-analytic functions that satisfy $\|g(z)\|>M(r, g)[\nu(r)]^{-\frac{1}{4}+\delta}$ have the asymptotic behavior

$$
g^{(m)}(z)=\left(\frac{\nu(r)}{z}\right)^{m}(1+o(1)) g(z) .
$$

In the Clifford analysis setting one thus obtains a similar asymptotic result when substituting the complex operator $z \frac{d}{d z}$ by the higher dimensional Euler operator $E$.

Acknowledgement. The authors are very thankful to Dr. Denis Constales (Ghent University) and to Prof. Gerhard Jank (RWTH Aachen) for a number of very fruitful discussions.

\section{References}

[1] Abul-ez, M. A. and D. Constales: On the order of basic series representing Clifford-valued functions. Appl. Math. Comput. 142 (2003)(2-3), 575 - 584.

[2] Brackx, F., Delanghe, R. and F. Sommen: Clifford Analysis. Research Notes in Mathematics 76. Boston: Pitman 1982.

[3] Constales, D. and R. S. Kraußhar: Representation formulas for the general derivatives of the fundamental solution of the Cauchy-Riemann operator in Clifford Analysis and Applications. Z. Anal. Anwendungen 21 (2002)(3), $579-597$. 
[4] Delanghe, R.: On regular points and Liouville's theorem for functions with values in a Clifford algebra. Simon Stevin 44 (1970/71), 55 - 66.

[5] Delanghe, R., Sommen, F. and V. Souček: Clifford Algebra and Spinor Valued Functions. Dordrecht: Kluwer 1992.

[6] Fueter, R.: Die Singularitäten der eindeutigen regulären Funktionen einer Quaternionenvariablen I. Comment. Math. Helv. 9 (1936)(1), 320 - 334.

[7] Fueter, R.: Funktionentheorie im Hyperkomplexen (Lecture notes written and supplemented by E. Bareiss, Fall Semester 1948/49). Math. Inst. Univ. Zürich 1949.

[8] Gürlebeck, K. and A. Hommel: On exponential functions in Clifford analysis (to appear).

[9] Gürlebeck, K. and W. Sprössig: Quaternionic and Clifford Calculus for Physicists and Engineers. Chichester: John Wiley \& Sons 1997.

[10] Jank, G. and L. Volkmann: Einführung in die Theorie der ganzen und meromorphen Funktionen mit Anwendungen auf Differentialgleichungen. UTB Wissenschaft. Basel: Birkhäuser 1985.

[11] Hayman, W. K.: The local growth of power series: a survey of the WimanValiron method. Can. Math. Bull. 17 (1974), 317 - 358.

[12] Hempfling, T. and R. S. Kraußhar: Order theory for isolated points of monogenic functions. Archiv Math. 80 (2003)(4), 406 - 423.

[13] Kraußhar, R. S.: Eisenstein Series in Clifford Analysis. Aachener Beiträge zur Mathematik 28. TH Aachen 2000 (Diss.).

[14] Kraußhar, R. S.: On a new type of Eisenstein series in Clifford analysis. Z. Anal. Anwendungen 20 (2001)(4), 1007 - 1029.

[15] Nevanlinna, R.: Zur Theorie der meromorphen Funktionen. Acta Math. 46 (1925), $1-99$.

[16] Valiron, G.: Lectures on the General Theory of Integral Functions. New York: Chelsea 1949.

[17] Wiman, A.: Über den Zusammenhang zwischen dem Maximalbetrage einer analytischen Funktion und dem größten Gliede der zugehörigen Taylorschen Reihe. Acta Math. 37 (1914), 305 - 326.

Received 11.11.2004 